\title{
Dissimulation au Service de la Providence dans Esther de Jean RACINE
}

\author{
Záviš ŠUMAN \\ Université Charles, Prague
}

\begin{abstract}
En): The article deals with persuasion in Racine's biblical tragedy Esther. It provides especially an analysis of rhetorical proofs as they are used in Esther's final récit (III, 4). We aim to demonstrate that the rational argumentation has a key role in this religious play. Despite the fact that many of the scenes might be understood in reference to biblical verisimilitude or to the concept of «merveilleux chrétien » and even if the visual appearence of Esther has a particularly strong impact upon Assuérus' action, the Racinian use of rhetoric reinforces the theatricality of the play.
\end{abstract}

Keywords (En): Jean Racine ; biblical tragedy ; rhetoric ; persuasion

Mots-clés (Fr) : Jean Racine ; tragédie biblique ; rhétorique ; persuasion

Je voudrais commencer par un constat simple: je ne suis pas le premier à remarquer que le thêâtre racinien est peuplé de personnages qui dissimulent, de façon qu'on puisse avoir l'impression que la dissimulation où le protagoniste ne lève pas le voile et où il n'agit pas à découvert soit l'essence même de la tragédie racinienne. Une consultation cursive de la concordance du théâtre et des poésies de Racine $^{1}$ ainsi que les études consacrées à l'usage racinien des sophismes ${ }^{2}$ nous confirment aussitôt dans cette intuition. Cette étude se propose d'effectuer l'analyse rhétorique du dispositif oratoire dans la tragédie biblique Esther. ${ }^{3}$

Pour illustrer l'omniprésence de la dissimulation chez Racine, je me bornerai ici à rappeler la tragédie romaine Bérénice où Titus, empereur de Rome, est contraint par la sévérité des lois de se séparer de la reine, sans cesser de l'aimer. Les atermoiements de Titus engendrent maintes dissensions et soupçons quant à la véritable intention du roi. Parmi d'autres occurrences notoires du faux-semblant, il convient de citer la longue exposition au cours des deux premiers actes de Phèdre dont la dramaturgie roule tout entière sur les aveux successifs des protagonistes. Or, l'exemple le plus connu sans doute, d'autant plus que Racine aura repris la même stratégie du dispositif scénique dans Esther, sans en tirer toutefois le même profit, est la scène où Néron épie Junie au cours de son rendez-vous avec Britannicus. Cette circonstance réduit Junie à dissimuler son amour pour

\footnotetext{
${ }^{1}$ Voir FreEMAN (1968).

2 Voir DECLERCQ (2001), HAWCROFT (2009).

${ }^{3}$ Cette étude fait partie du projet GAČR 16-08622S Raně novověká francouzská tragédie: pnutí mezi teorií a praxí. Nous citons Esther dans l'édition de Georges FORESTIER (RACINE, 1999). Il va sans dire que notre analyse ne reste que partielle. Pour une interprétation synthétisante, il faudrait mesurer notamment le rôle dramaturgique des séquences chantées et de la musique. Sur l'imbrication (et non pas une simple insertion) du chant et de la musique et des parties déclamées dans Esther, voir Louvat-Molozay (2003), Piejus (1997), Woshinsky (1994). Sur les nombreuses adaptations du Livre d'Esther aux seizième et dix-septième siècles en France, voir MASTROIANNI (2001).
} 
Britannicus car elle n'ignore pas, à la différence de ce dernier, la présence indiscrète de l'empereur. Racine se plaît ainsi à dédoubler le rôle du spectateur : en fait Néron, en tant que personnage muet, incarne l'idée du théâtre dans le théâtre.

Ces quelques exemples suffisent, je l'espère, pour voir que la dissimulation ainsi que la simulation sont pour Racine garantes de l'efficacité dramatique et du suspense. De surcroît, les scènes de dissimulation, appuyées dans Britannicus par le dédoublement du rôle du spectateur évoqué ci-dessus, offrent un terrain d'élection pour les analyses de l'imbrication de l'action représentée avec le mécanisme de l'adhésion au spectacle. Autrement dit, elles décèlent une dimension métathéâtrale car elles mettent en jeu divers procédés de persuasion mis à l'œuvre et que le dramaturge exploite avec subtilité afin de susciter le plaisir esthétique. Ce rôle de la dissimulation, voire de la simulation au second degré ne va pas, dans un certain sens, sans paradoxe. En effet, selon les revendications de certains théoriciens qui visent à ce que la représentation théâtrale devienne, par une sorte d'agréable mensonge, une part de la réalité vécue, le spectateur ne serait qu'un témoin oculaire et muet de l'action qu'il est pourtant censé percevoir comme véritablement advenue, tout comme s'il en était l'acteur. De plus, il est souhaitable qu'il en soit dupe car il est amené à ne pas mettre en doute, de façon certes passagère et seulement le temps de la représentation, ce qu'il voit et ce qu'il entend. Nous pourrions donc en inférer que les dissimulations des personnages raciniens concourent à ne pas entraver le bon fonctionnement du mécanisme de l'illusion mimétique, comme si le déploiement manifeste des enjeux qui président à la dis/simulation de l'action représentée possédait la vertu de faire oublier au spectateur, sur-le-champ et momentanément, qu'il est, lui aussi, l'objet d'une manipulation.

Avant d'entamer l'analyse du dispositif oratoire dans Esther, tâchons d'esquisser, sans prétendre à l'exhaustivité, une typologie de dissimulation/s telle qu'elle est à l'œuvre dans cette tragédie biblique.

\section{Lieu territorialisé et rouages de l'action}

Le Palais d'Assuérus où se déroule l'action de la pièce est un lieu mystérieux, cloisonné et fortement territorialisé. Racine ne respecte pas d'ailleurs ce qu'on appelle l'unité exacte de lieu : le décor change et la pièce se termine dans des lieux contigus qui permettent facilement la rencontre des personnages qui s'opposent. En particulier la chambre d'Assuérus, où se déroule l'acte II de la pièce, est un huis clos où même la reine ne peut entrer sans l'autorisation du roi. Seuls les conseillers les plus proches d'Assuérus y ont accès. Mais c'est un accès policé, ce qui en renforce la dimension tragique. Esther qui ne peut pas approcher le roi à son gré évoque la chambre d'Assuérus à l'aide de l'un des topoï liés à la dissimulation, à savoir l'opposition entre le visible et l'invisible qui se double de celle de la présence et de l'absence. À l'injonction de Mardochée de découvrir au roi sa véritable identité afin d'empêcher l'extermination des Juifs, Esther répond: «Hélas! Ignorez-vous quelles sévères lois / Aux timides mortels cachent ici les Rois ? / Au fond de leur Palais leur majesté terrible, / Affecte à leurs Sujets de se rendre invisible.»(I, 3, v. 191-194; nous soulignons.) Loin de toute 
indétermination, l'espace ainsi présenté est non seulement propice à accroître le tragique de la pièce mais l'ancrage spatial favorise également les rebondissements de l'intrigue. Hydaspe, en tant qu'adjuvant d'Aman, croit pouvoir déceler les mystères du Palais en exploitant la même dialectique entre le visible et l'invisible : « Je me souviens toujours que je vous ai juré / D'exposer à vos yeux par des avis sincères / Tout ce que ce Palais renferme de mystères. » (II, 1, v. 380-382; nous soulignons.) Or, comme cette affirmation s'avèrera vaine, elle relève de l'ironie tragique, à plus forte raison qu'Aman reste convaincu qu'Hydaspe voit tout ce qui se passe dans le Palais (cf. «Toi, qui dans ce Palais vois tout ce qui se passe... », II, 1, v. 442). Cette confiance contraste avec l'attitude beaucoup plus circonspecte des jeunes filles israélites. Au milieu du chœur qui clôt le second acte, l'une d'elles fait preuve d'une véritable prudence. S'adressant à ses jeunes compagnes, elle les enjoint de baisser la voix : «Parlons plus bas, mes Sœurs. Ciel! si quelque Infidèle / Écoutant nos discours nous allait déceler !» (II, 8, v. 750-51) Élise, confidente d'Esther, reprend cependant la peureuse et l'incite à ne rien craindre, situation dramatique qui répond exactement à la même réfutation d'Assuérus dans la troisième scène du premier acte où la reine évoque les obstacles qui l'empêchent d'accéder à la chambre du roi. L'ambiguïté morale de cet espace éminemment tragique nous amène à une dernière observation: renforçant les liens entre l'espace et l'action, la dramaturgie racinienne permet d'intégrer à la pièce non seulement les très nombreuses considérations habituelles sur la déchéance morale de la cour pervertie et rompue à l'affectation, mais aussi d'introduire le personnage traditionnel dans le personnel tragique, à savoir le mauvais conseiller, incarné ici par Aman.

C'est au début de l'acte III que la dissimulation devient le ressort dramaturgique par excellence, ce dont témoignent les propos machiavéliques et sentencieux de Zarès, femme d'Aman, qui donne à son mari une véritable leçon de morale arriviste : «Dissimulez, Seigneur, cet aveugle courroux. » (III, 1, v. 831) ; «Quiconque ne sait pas dévorer un affront, / Ni de fausses couleurs se déguiser le front, / Loin de l'aspect des Rois qu'il s'écarte, qu'il fuie. / Il est des contretemps qu'il faut qu'un Sage essuie. / Souvent avec prudence un outrage enduré / Aux honneurs les plus hauts a servi de degré. » (III, 1, v. 838-843) Ses conseils font contraste avec le chant non moins sentencieux des jeunes filles israélites (cf. III, 2, v. 969-84, 987-988, 999-1005). Celui-ci balise à son tour le terrain au réquisitoire d'Esther développé dès lors qu'elle aura révélé sa véritable identité à Assuérus. À mesure que la pièce progresse, les secrets et les machinations se dissipent. Ce n'est pas seulement la suavité du chant des jeunes Israélites et l'ingéniosité discursive du réquisitoire final de la reine, mais aussi la disposition scénique. Les jardins d'Esther, baignés de lumière, renforcent l'opposition entre l'intérieur et l'extérieur et tous ces éléments favorisent ensemble le lever du voile et détrompent peu à peu le roi trop crédule et jusque-là aveuglé.

\section{Dénouement : dévoilement progressif de la vérité}

Venons-en maintenant à l'analyse plus pointue de ce «déssillement» (cf. le vers prononcé par Assuérus : «Mes yeux sont dessillés, le crime est confondu. » 
III, 7, v. 1178) qui n'a lieu qu'à l'extrême fin de la tragédie. Comment est-il préparé ? Répond-il aux critères de la « vraisemblance biblique »? Quel est le rôle du chœur dans les scènes qui le précèdent ? Pourquoi la stratégie argumentative déployée par Esther est-elle efficace alors que celle d'Aman échoue nonobstant le fait que les deux relèvent, au départ, d'une stratégie dissimulatrice ?

L'exposition de la pièce nous fait assister à l'entrevue entre Esther et Élise, sa confidente. Longtemps sans nouvelles de la reine, Élise s'enquiert de «secrets ressorts » (I, 1, v. 29) qui l'ont hissée sur le trône persan. Esther lui fait part ensuite de la répudiation de l'altière Vasthi, ancienne femme d'Assuérus, et de l'intention de Mardochée, son oncle qui l'a élevée, de rétablir les droits des Juifs grâce au mariage avec Assuérus. Or quoiqu'Esther ne sût rien des «desseins secrets » (I, 1, v. 53) de Mardochée, elle s'y plie. Dès le début de l'exposition, nous apprenons qu'Esther n'a pas encore révélé sa vraie identité au roi. Racine se ménage cette occasion pour introduire un passage sur l'omniprésence de l'esprit de parti et d'une séduction frivole dont :

\section{ESTHER}

Je vins. Mais je cachai ma race et mon pays.

Qui pourrait cependant t'exprimer les cabales,

Que formait en ces lieux ce peuple de Rivales,

Qui toutes disputant un si grand intérêt,

Des yeux d'Assuérus attendaient leur arrêt?

Chacune avait sa brigue et de puissants suffrages.

L'une d'un sang fameux vantait les avantages.

L'autre pour se parer de superbes atours,

Des plus adroites mains empruntaient le secours.

Et moi, pour toute brigue, et pour tout artifice,

De mes larmes au Ciel j'offrais le sacrifice. (I, 1, v. 54-64 ; nous soulignons.)

Or, comme souvent chez Racine et en dépit de divers modes de l'esthétique tragique qu'il pratiquait, les larmes servent ici moins de moyen de persuasion que d'attraction, voire d'un certain envoûtement : pour preuve, la scène centrale du second acte où la reine entre dans la chambre du roi. Loin de la répudier, Assuérus est attendri et semble prêt à tout lui accorder. De fait, Esther, en tant qu'instrument du Dieu omnipotent (cf. I, 1, v. 67 : « Dieu tient le cœur des Rois entre ses mains puissantes. »), a été choisie par Assuérus pour être promue à la dignité du rang royal, et la fréquentation de la cour semble l'avoir vite initiée à l'art de la dissimulation, dissimulation «honnête » peut-on ajouter en allusion au célèbre traité de Torquato Accetto qui consacre tout un chapitre à la dissimulation servant la piété parentale. ${ }^{4}$ Cultivant son ethos de femme modeste et consciente - la reine a « une dimension quasi messianique », dit FORESTIER (in RACINE 1999 : 1696) de l'état du délabrement du Temple juif (I, 1, v. 81-88), Esther continue à se taire sur sa véritable identité. Tout comme dans Iphigénie, le sujet de la pièce - à savoir la révélation de l'identité juive de la reine qui débouchera in extremis sur la clémence d'Assuérus et, par conséquent, sur le rétablissement des Juifs - est tiré

\footnotetext{
${ }^{4}$ Sur la réception du traité d'Accetto en France, voir VAN DeLFT (1993). Voir aussi l'introduction de Salvatore S. Nigro à la traduction française moderne (NIGRO in ACCETTO 1989 : 7-17).
} 
du « fond de la tragédie » (cf. la préface de Racine à Iphigénie). On peut même dire que, vu son aspect providentiel, cette tragédie biblique, qui n'est pas dépourvue d'un certain manichéisme, en particulier dans des séquences déclamées ou chantées qui incombent au chœur, repose en grande partie sur l'atermoiement de l'aveu de son identité, car la résolution en fut prise par Esther avec plus de promptitude qu'il ne lui en faut pour la mettre à exécution. Aussi bien que dans Bérénice, le spectateur s'intéresse avant tout, et malgré quelques coups de théâtre, à la stratégie persuasive de la reine.

J'ai déjà évoqué la nature hermétique, spatialement close du Palais royal d'Assuérus. Elle l'est mais paradoxalement cela n'empêche pas l'arrivée inattendue de Mardochée dans la chambre de la reine. G. Forestier fait remarquer que cette entrée en scène de Mardochée sombre, revêtu d'un silice et couvert de cendre, pèche contre la vraisemblance et ne saurait être expliquée que dans le régime du merveilleux chrétien (cf. le commentaire de Forestier, p. 1696-1697). En effet, Esther s'étonne de la présence de Mardochée : «Un Ange du Seigneur sous son aile sacrée / A donc conduit vos pas, et caché votre entrée ? (I, 3, v. 157-158), bien qu'elle fût «préparée » de façon, il est vrai, on ne peut plus vague (cf. «Absent je le [Mardochée] consulte. Et ses réponses sages / Pour venir jusqu'à moi trouvent mille passages. » I, 1, v. 95-96). Dès lors, on peut affirmer que la logique interne du développement de l'intrigue obéit moins au régime de la vraisemblance au sens strict du terme qu'à une logique providentielle qui permet aux deux protagonistes de se rencontrer, de se parler à découvert et ainsi de nouer une véritable action théâtrale. Mardochée ne peine pas trop à persuader la reine d'agir et de sauver les Juifs contre les complots d'Aman qui demande qu'on les extermine.

À l'image de la loi sévère qui interdit l'accès à Assuérus, évoquée par Esther (I, 3, v. 191-204), répond une éthopée du deus terribilis de l'Ancien Testament («Dieu fort et jaloux », Exode XX, 5) dont les véritables intentions restent cachées aux faibles humains. Mardochée en donne une preuve lorsqu'il s'adresse à Esther : «Et qui sait, lorsqu'au trône il conduisit vos pas, / Si pour sauver son peuple il ne vous gardait pas ? (I, 3, v. 211-212) La modalité hypothétique de l'affirmation de Mardochée est en réalité une injonction : tout en recourant aux lieux communs issus de la topique rhétorique, le discours de Mardochée roule avant tout sur la présupposition de la stricte obéissance au Dieu omnipotent dont les intentions sont mues par la logique de la grâce efficace. Ainsi s'instaure dans la tragédie une opposition nette entre le sacré providentiel et le régime profane. Si la dimension profane est dominée par les forces dissimulatrices telles que les représentent la cour hypocrite et ses intrigues, calomnies, brigues, complots et silences, ${ }^{5}$ on ne peut pas dire que la dimension sacrée en soit dépourvue : cela dit, la dissimulation entre ici au service de la providence qui seule permettra que l'aveuglement des crédules, victimes de duplicité, cède le pas au rayonnement de la vérité, comme si le but sacrait les moyens.

Arrivés au dénouement de la tragédie, voyons comment Esther procède pour empêcher l'extermination des Juifs. Son apparition finale - telle une épiphanie -

${ }^{5}$ Voir ReVaz (1998 : 220). 
est soigneusement préparée. On sait qu'Assuérus succombe facilement à son charme comme on l'apprend déjà à l'acte II où le roi évoque sa beauté par le biais $\mathrm{du}$ «je ne sais quoi » ainsi que du vocabulaire issu de la tradition du sublime. De surcroît, la scène de la révélation finale succède au chœur, en partie chanté, en partie déclamé, qui commente tantôt de façon explicite tantôt par des allusions allégoriques ce que les filles sont en train de voir. Tandis que la séquence déclamée, qui consiste principalement en une éthopée d'Aman approchant la salle où se tient le festin d'Esther, fait de nouveau usage de la dialectique entre le visible et l'invisible (III, 3, v. 934-941), le passage chanté articule les éloges du bon monarque avec tout un faisceau de qualités requises : générosité, justice, et surtout le sens du discernement (III, 3, v. 955-1015). Tout particulièrement les quatre stances chantées alternativement par une voix seule et par tout le chœur (III, 3, v. 969-984) tournent autour des calomnies et de la duplicité des imposteurs qu'un roi juste devrait savoir discerner. On ne sait pas exactement si Assuérus entend/écoute tout ce que chante le chœur, mais s'il en est ainsi, on peut interpréter les mélodies chantées comme une amorce de la stratégie persuasive que reprendra le réquisitoire d'Esther à la scène de la révélation. ${ }^{6}$

En revanche, l'on sait avec certitude qu'Aman, quant à lui, assiste à la cérémonie et on est en bon droit de supposer que l'éloge du bon monarque doit l'inquiéter d'autant qu'il a cru pouvoir saisir la vraie signification du songe prémonitoire ${ }^{7} \mathrm{~d}^{\prime}$ Assuérus interprété par les savants chaldéens. Le motif du rêve peut, lui aussi, nourrir une réflexion dans le cadre de notre sujet. Le moment où Hydaspe l'évoque (II, 1, v. 383-384) est plutôt éloigné de celui où l'on en apprend le sens apparent (III, 2, v. 921-925). À part de servir le suspense et donc la dramaticité de la pièce, le rêve y réinsère le problème de la dualité entre l'être et le paraître par l'intermédiaire du rapport entre le sens manifeste et le sens latent/caché, autrement dit le problème de la vérité et des modalités de son apparition. Le délai qui sépare ce signe prophétique de son déchiffrement en dit long sur le parcours sinueux qui va de l'apparence, du faux/vrai-semblant au vrai, de la dissimulation à l'aveu. Or la supposition d'Aman d'avoir compris la signification du songe d'Assuérus se verra encore démentie. Il s'agit en fait d'un autre coup de théâtre, relevant de l'ironie tragique, et qui fait de plus un pendant à la scène la plus réussie de l'acte précédent (II, 5) où Assuérus s'enquiert auprès d'Aman des moyens de récompense d'un sujet fidèle. Aman, aveuglé par l'ambition et faute de discernement, se croit l'objet de l'honneur et peint en vives couleurs la cérémonie envisagée. Plus il surenchérit sur son faste, plus cuisants seront l'humiliation et l'échec qu'il essuiera comme le confirmera la scène 4 de l'acte III qui réunit Assuérus, Esther, Aman, Élise et le chœur. C'est Assuérus qui prend l'initiative de la parole. Il s'extasie tant sur les « grâces secrètes » (v. 1016) des discours d'Esther que sur la «noble pudeur» (v. 1017) de ses actions. Ces vertus qu'il estime par-dessus tout éveillent en lui la curiosité quant à l'origine de la reine. Assuérus qui cultive son ethos du roi amoureux et généreux convie Esther

\footnotetext{
${ }^{6}$ Les scènes 3 et 4 de l'acte III s'enchaînent par une liaison de vue.

${ }^{7}$ Sur la fonction dramaturgique du songe d'Assuérus, élément de l'invention racinienne, voir BLANC (1998: 20-21).
} 
à parler. La modeste Esther se prosterne devant lui et l'implore pour sa propre vie et pour celle des Juifs. Le roi ne comprend ni la requête ni l'aveu voilé d'Esther car ils ont la forme d'un enthymème (forme réduite du syllogisme dont la prémisse mineure serait « Je suis Juive. »), et il la relève immédiatement. C'est à ce moment qu'Esther dévoile ses racines juives, cette fois-ci de façon directe et on ne peut plus cursive, et en outre devant les témoins, y compris le perfide Aman qui interrompt deux fois cette séquence initiale de la scène de l'aveu. Elle ne manque pas non plus de signaler l'édit royal ordonnant l'extermination des Juifs. La mention de l'édit en vigueur, donc le recours à une preuve objective extrinsèque, corrobore l'urgence tragique de la situation. Ceci dit, jusqu'ici la charpente de la scène répond surtout aux besoins dramaturgiques alors qu'à partir de ce moment elle annexe puissamment les moyens rhétoriques, notamment ceux qui appartiennent au genre judiciaire. La réplique d'un Assuérus étonné qui consiste en un syllogisme complet en modalité dubitative exploite le lieu commun des contraires et finit par la mise en doute de la conséquence en apparence logique du raisonnement (III, 3, v. 1036-1039). Si l'on essaie de reconstituer la chaîne argumentative du roi, on obtient comme proposition mineure Les Juifs descendent d'une source impure, comme proposition majeure Or Esther est sage et innocente, et comme conclusion Esther, serait-elle Juive? En réalité, le raisonnement d'Assuérus est encore plus complexe, non seulement parce que la conclusion revêt une forme hypothétique qui laisse entrevoir une possible faille dans l'argumentation (non-validité de la proposition mineure), mais aussi parce qu'elle se greffe sur l'implication d'une seconde proposition mineure qui serait J'aime Esther. Il s'agit en fait d'un double enthymème.

Aussi dans le très long récit qui suit (v. 1044-1135), Esther entreprend-elle surtout la réfutation de la première proposition mineure du raisonnement d'Assuérus. Sa complexité n'en diminue pas l'efficacité, comme si le Dieu auquel Esther s'adresse à la fin de la quatrième scène du premier acte avait réellement prêté à son discours « le charme » (I, 4, v. 290) qu'elle avait sollicité. Du point de vue dramaturgique, le récit d'Esther a pour fonction de prévenir non seulement le danger imminent de la mort de Mardochée, mais aussi l'extermination des Juifs qu'Esther présente comme un peuple soumis au pouvoir légitime du roi perse.

Dans la partie suivante, nous essaierons de dégager les procédés que la reine met en œuvre dans sa stratégie discursive complexe qui tient à la fois aux genres judiciaire (réquisitoire contre Aman) et épidictique (éloge voilé du roi juste). La longueur de la séquence respective (III, 4, v. 1044-1140) ne nous permet malheureusement pas de la citer ici in extenso. Pour tirer un meilleur profit de notre analyse, le lecteur est prié de se reporter à l'édition de G. FoRESTIER (RACINE, 1999).

\section{Structure rhétorique du réquisitoire d'Esther}

Le discours d'Esther se déroule en plusieurs étapes. S'étant assuré les bonnes grâces du roi (ethos/pathos, captatio benevolentiae), Esther invoque brièvement Dieu (v. 1044). Réitérant sa prière, elle cultive son ethos de femme sincère, 
modeste et animée d'une profonde foi. L'exorde contient ensuite une courte proposition (v. 1045-1049) dans laquelle la reine exploite habilement les lieux communs intrinsèques de circonstances, de contraires et de cause/conséquence, et s'inscrit en faux contre la conviction d'Assuérus que les Juifs sont «le rebut des humains » (v. 1046). La faveur de Dieu pour un peuple pieux, fidèle et respectueux de l'héritage qui lui incombe en est une preuve objective qu'Esther développe dans une éthopée célébrant l'omnipotence d'un Dieu sévère mais équitable. Le récit exploite une nouvelle fois les lieux communs intrinsèques qui se greffent sur une série d'antithèses entre le pouvoir divin et le pouvoir séculier, et entre la pérennité de l'un et la précarité de l'autre. Cette séquence épidictique (v. 1050-1057) pourrait être interprétée comme une digression à la narration d'autant plus que le vers 1050 est, dans la première édition de la pièce, mis en retrait par un nouvel alinéa. L'opposition signalée ci-dessus réapparaît avec le retour à la narration car la relation du passé du peuple juif met en relief sa prospérité lorsqu'il est demeuré fidèle à son Dieu et au contraire son infortune lorsqu'il s'en est détourné. Cet éloge de la justice divine s'intègre étroitement non seulement à cette relation, mais il prépare le terrain pour l'introduction du portrait de Cyrus Le Grand (v. 1062-1073), roi exemplaire de la Perse agissant en accord avec la providence divine. Le récit d'Esther se fonde ainsi sur le règne du plus glorieux roi qui mit fin à la captivité de Babylone et reconstitua le culte religieux. Même si la narration qui dépeint les hauts faits de Cyrus s'apparente là encore au genre épidictique, sa véritable fonction est tout autre. S'appuyant sur l'induction à savoir sur l'analogie implicite tirée de l'exemple particulier - Esther suggère au roi d'assurer la continuité brisée par le roi Cambyse, « héritier insensé » de Cyrus. L'éloquence encomiastique d'Esther célébrant un roi juste entre ici au service de la démarche persuasive subtilement masquée. Arrivée à l'époque actuelle du règne d'Assuérus, Esther exploite les preuves subjectives, l'ethos et le pathos. Le contenu propositionnel du discours sentencieux qui s'enchaîne à l'éloge du roi juste (v. 1080), celui de la pitié de Dieu avec les malheureux (v. 1079), prend la forme des enthymèmes que nous avons déjà signalés et que l'on pourrait facilement reconstituer étant donné que ce discours tisse bien sûr un lien analogique entre les deux objets de l'éloge devenus un lieu de comparaison récurrent. Il permet en outre d'apporter un nouvel élément à la réfutation visant à rectifier la présupposition initiale d'Assuérus quant à l'impureté de la race juive. De même que l'exemple qui a recours à l'amplification dans la narration des faits glorieux de Cyrus (v. 1065-1073), la brièveté des sentences et leur force persuasive servent le projet délibératif de la reine. En esquissant une image flatteuse d'un roi juste et en l'opposant dans ce qui suit à la cruauté d'un Aman perfide, Esther s'assure la bienveillance du roi.

À partir de ce moment, le discours de la reine relève principalement du genre judiciaire. L'hypotypose prospective (v. 1083-1085, v. 1098-1103) ${ }^{8}$ se voit plusieurs fois interrompue par le réquisitoire pendant lequel Aman se déclare malgré lui ce qui prouve d'ailleurs qu'il n'a pas su mettre à profit les conseils machiavéliques de sa femme, et donc qu'il n'est pas, contrairement à Esther,

\footnotetext{
${ }^{8}$ Voir DECLERCQ (2001).
} 
maître de son ethos. La fonction argumentative du procédé en question consiste donc à mettre en garde contre les manipulations d'Aman, nommément identifié. En peignant les conséquences présumées que pourrait entraîner le pouvoir débridé du «perfide Étranger » (v. 1101) et en assignant le succès des conquêtes politiques et territoriales d'Assuérus au Dieu des siens, Esther en tant que porte-parole du peuple juif opprimé crée un rapport de connivence avec Assuérus. Les deux partis sont désormais soudés non seulement parce qu'ils professent le même respect de la vertu, mais aussi parce qu'ils font face au même danger, à cette différence près que celui-ci est hypothétique pour Assuérus, mais imminent pour Esther et Mardochée.

Dès lors, Esther peut reconstituer les reproches qui pèsent sur les Juifs dans une subjectio amplifiée construite en isocolon (v. 1105-1106), et les réfuter l'un après l'autre au moyen des preuves extrinsèques. Esther fait ainsi défiler dans le plaidoyer pour son peuple les complots des intrigants, la loyauté des Juifs vis-à-vis d'Assuérus manifeste dans leurs prières pour sa prospérité, l'extension du territoire de la Perse, et surtout l'échec de l'attentat contre Assuérus. La reine les met en avant à titre d'interventions divines par l'intermédiaire des Juifs, peuple soumis et à Dieu et au pouvoir royal légitime. L'autorité de la parole divine dont Esther est dépositaire et dont elle paraît nimbée se transforme ici en une évidence irréfutable de sorte que la péroraison, partie de la dispositio incontournable dans le genre judiciaire et que Racine exploite souvent dans ses tragédies profanes, puisse faire défaut. À peine Esther fait-elle l'aveu de son identité et du lien de parenté qui l'unit à Mardochée, qu'elle confirme la malhonnêteté d'Aman qui subit le sort fréquent du traître qui se trahit lui-même à son corps défendant et à son insu.

Dans cette situation, le tragique de la pièce éclate avec force car l'unité de péril devient évidente aussi pour Assuérus et elle s'inscrit dans un mouvement diphasé où, comme on verra, les ressorts de persuasion changent de nature. D'une part, le régime de l'évidence de la parole révélée, qui anticipe la décision finale du roi, est constamment à l'œuvre, certes, mais d'autre part on ne saurait sous-estimer la stratégie argumentative de la reine. Celle-ci donne du poids à son procès à charge d'Aman en éclaircissant les motifs de son comportement : elle dévoile au roi l'orgueil, la rancune, l'excès d'ambition et la susceptibilité de son conseiller qui va de pair avec son goût de la vengeance. Esther surenchérit donc en ajoutant aux preuves qu'elle a fournies des éléments de circonstance d'un autre ordre qui devraient conduire le roi à réaménager le champ du faux et du vrai, tâche difficile et menacée de déni lorsqu'ils sont en rapport d'opposition. Ces éléments stimulent alors le mouvement d'adhésion à la vérité car ils renforcent sa vraisemblance via le lien, appréhendé comme causal, entre les mœurs d'un personnage et ses actes. Cependant Racine construit l'intrigue de sorte de prolonger aussi longtemps que possible le suspense dramatique : Aman se voit démasquer par Esther, mais le roi ne lui donne pas l'occasion de se défendre. Le récit d'Esther suffit, en effet, à le persuader. Comme on a signalé ci-dessus, Assuérus est détrompé et la simple vue d'Aman à genoux devant Esther le convainc de la véridicité des paroles de la reine (cf. III, 6, v. 1169-1171 : «Dans ses yeux [d'Aman] confus je lis ses perfidies / Et son trouble appuyant la foi de vos discours, / De tous ses attentats me rappelle le cours. »; v. 1178 «Mes yeux sont dessillés, le crime est confondu.»; nous 
soulignons). La stratégie persuasive d'Esther, telle une phase préparatoire, a frayé la voie à la force de conviction d'un ordre supérieur, à savoir celle de l'instance divine (cf. la scène finale, v. 1227 : «Elle a parlé, le Ciel a fait le reste. ») garante de la vérité, qui semble éclipser la parole profane et les dispositifs rhétoriques traditionnels. ${ }^{9}$

Il va sans dire que cette armature rhétorique se voit étayée par d'autres phénomènes textuels, dont les modes d'expression de la cause. On remarque d'abord la multitude de ce que l'on pourrait appeler les micro-séquences causales dans lesquelles le rapport de causalité est « déguisé » en rapport de temps (cf. p. ex. v. 1047-1049) ou privé de connecteurs logiques et rabattu donc à la simple juxtaposition (cf. p. ex. v. 1058-1059). La causalité ainsi escamotée - masquée ou reléguée à l'implicite - participe du jeu de dissimulation de l'ensemble et insère dans la structure du logos de menues zones d'indétermination d'où ressort cependant le pouvoir de la providence divine. Deuxièmement, les mots placés à la rime ainsi que les allitérations en $« \mathrm{r} »$ (p. ex. v. 1062, 1097, etc.) renforcent les procédés argumentatifs associés aux actes de violence et aux conflits entre la justice et l'injustice. L'analyse approfondie du plan de l'expression pourrait démontrer, dans le prolongement des travaux de P. FRANCE (1965), M. HAWCROFT (1992, 2009) et G. DECLERCQ (1984, 2001), que l'elocutio racinienne et même les jeux du rythme et de la structuration de rimes sont, eux aussi, au service de l'argumentation.

En guise de conclusion nous pourrions dire que la dissimulation obéit dans cette tragédie à un double régime. Premièrement, Racine s'en sert pour assurer à la pièce la dramaticité et un vrai suspense où rien n'est joué à l'avance. Deuxièmement, elle permet de mettre en avant la providence divine et son rôle en introduisant l'opposition radicale entre l'ordre profane et l'ordre sacré. Contrairement à ce que l'on pourrait croire, ces deux ordres ne coïncident pas l'un avec le pôle moral de la franchise et l'autre avec le pôle immoral de la dissimulation. Comme j'ai tâché de démontrer, la tragédie et surtout son dénouement font voir que malgré le régime de providence, ${ }^{10} l^{\prime}$ hérö̈ne déploie une stratégie discursive qui se fonde en grande partie sur le logos, c'est-à-dire sur les arguments rationnels. Sans que la suprématie du sacré en pâtisse, le sublime soustendu par le merveilleux chrétien et cautionné par l'autorité supérieure de Dieu daigne mettre à profit les moyens que la rhétorique met à sa disposition, bien qu'ils appartiennent au profane. Cette tragédie biblique répond, somme toute, à un schéma double où la coexistence des deux régimes savamment articulés obéit au dessein dramaturgique de Racine : faire du thêatre, c'est faire parler et faire voir. ${ }^{11}$

\footnotetext{
${ }^{9}$ Certains critiques mettent en cause le tragique des pièces bibliques, et a fortiori celui des tragédies chrétiennes. Nous ne souscrivons pas à cette vue car elle nous paraît négliger tant les aspects politiques que les enjeux passionnels, bien présents dans Esther, voir MAZOUER (1992).

${ }^{10}$ Sur l'imbrication du profane et du sacré due en partie au traitement racinien en sources, voir SCHOLAR (2002).

${ }^{11}$ Rappelons la signification du verbe grec theaomai (regarder, observer). Sur l'enchevêtrement des éléments visuels et verbaux dans Esther et Athalie, voir MASKELL (1992).
} 


\section{BIBLIOGRAPHIE}

ACCETTO Torquato (1990), De l'honnête dissimulation, traduit de l'italien par M. BLANC-SANCHEZ, édition établie, annotée et introduite par Salvatore S. NIGRO, Paris, Verdier.

BLANC Emmanuèle (1998), D'Esther à Athalie : une forme à la recherche de son sens, L'Information littéraire 2, p. 20-25.

CAMPBEll John (2009), The Politics of Esther, Seventeenth-Century French Studies, Vol. 31, No. 1, p. 25-35.

DECLERCQ Gilles (1984), La Ruse oratoire dans les tragédies de Racine, Cahiers de littérature du XVII ${ }^{e}$ siècle 6, p. 115-123.

DECLERCQ Gilles (1992), L'art d'argumenter: structures rhétoriques et littéraires, Paris, Éditions Universitaires.

DECLERCQ Gilles (2001), Poéticité versus rhétoricité : pathos et logos dans les tragédies de Racine, in : Racine et/ou le classicisme, éd. R. W. TOBIN, Biblio 17-129, Tübingen, Gunter Narr Verlag, p. 19-53.

FRANCE Peter (1965), Racine's Rhetoric, Oxford, Oxford University Press.

FreEMAn Bryant C. (1968), Concordance du Théâtre et des Poésies de Jean Racine, 2 tomes, New York, Cornell University Press.

GIULIANI Pierre (2004), Esther et la douceur: une gageure dramatique, L'Information littéraire 4, p. 3-11.

HAWCROFT Michael (1992), Word as Action : Racine, Rhetoric, and Theatrical Language, Oxford, Clarendon Press.

HAWCROFT Michael (2009), La mauvaise rhétorique chez Racine, in: Racine poète, éd. B. LOUVAT et D. MonCONDHU'Y, Revue La Licorne 50, p. 355-368.

LOUVAT-MolOZAY Bénédicte (2003), Esther et Athalie, tragédies avec musique : Racine et la dramaturgie de l'introduction musicale, in : Jean Racine 16391699. Actes du colloque Île-de-France - La Ferté-Milon 25-30 mai, éd. G. DECLERCQ et M. Rosellini, Paris, Presses universitaires de France.

MASKELL David (1992), The hand of God in French religious drama: Racine, Boyer, and Campistron, Seventeenth-Century French Studies 14, p. 119-131.

MAZOUER Charles (1992), Les tragédies bibliques sont-elles tragiques?, Littératures classiques 16, p. 125-140.

MASTROIANNI Michele (2001), L'Esther francese : da figura a personaggio, in : Il tragico e il sacro dal Cinquecento a Racine, éd. D. CECHETTI et D. DALLA VALLE, Biblioteca dell'Archivum Romanicum, Leo S. Olschki Editore, p. $175-226$.

PIEJUS Anne (1994), La tragédie chrétienne: théâtre et musique à Saint-Cyr, Littératures classiques, 21, p. 139-148.

PIEJUS Anne (1997), Esther, un modèle paradoxal du théâtre musical pour SaintCyr, PFSCL, XXIV, 47, p. 395-420.

RACINE Jean (1999), Euvres complètes, I, Théâtre - Poésie, éd. G. FORESTIER, Paris, Gallimard, Bibliothèque de la Pléiade.

REVAZ Gilles (1998), La représentation de la monarchie absolue dans le théâtre racinien. Analyses socio-discursives, Paris, Éditions Kimé. 
SCHOLAR Richard (2002), «Je ne sais quelle grâce »: Esther before Assuérus, French Studies, vol. LVI, nº 3, p. 317-327.

VAN DELFT Louis (1993), T. Accetto et la notion de dissimulation honnête dans la culture classique, in: l'Honnête homme et le dandy, éd. A. MONTANDON, Tübingen, p. 35-57.

WOSHINSKY Barbara R. (1994), La musique parlante : la fonction du chœur dans l'Esther de Racine, Littératures classiques 21, p. 149-161. 